\title{
Post-discharge surveillance (PDS) for surgical site infections: a good method is more important than a long duration
}

M B Koek (mayke.koek@rivm.nl) ${ }^{1}$, J C Wille ${ }^{1}$, M R Isken², A Voss ${ }^{2,3}$, B H van Benthem ${ }^{1}$

1. Department of Epidemiology and Surveillance, Centre for Infectious Disease Control, National Institute for Public Health and Environment (RIVM), Bilthoven, the Netherlands

2. Medical Microbiology \& Infectious Diseases, Canisius-Wilhelmina Hospital, Nijmegen, the Netherlands

3. Department of Medical Microbiology, Radboud University Nijmegen Medical Centre, Nijmegen, the Netherlands

Citation style for this article:

Koek MB, Wille JC, Isken MR, Voss A, van Benthem BH. Post-discharge surveillance (PDS) for surgical site infections: a good method is more important than a long duration. Euro Surveill. 2015;20(8):pii=21042. Available online: http://www.eurosurveillance.org/ViewArticle.aspx?Articleld=21042

Article submitted on 18 August 2014 / published on 26 February 2015

Post-discharge surveillance (PDS) for surgical site infections (SSIs) normally lasts 30 days, or one year after implant surgery, causing delayed feedback to healthcare professionals. We investigated the effect of shortened PDS durations on SSI incidence to determine whether shorter PDS durations are justified. We also studied the impact of two national PDS methods (those mandatory since 2009 ('mandatory') and other methods acceptable before 2009 ('other')) on SSI incidence. From Dutch surveillance (PREZIES) data (19992008), four implant-free surgeries (breast amputation, Caesarean section, laparoscopic cholecystectomy and colectomy) and two implant surgeries (knee replacement and total hip replacement) were selected. We studied the impact of PDS duration and method on SSI incidences by survival and Cox regression analyses. We included 105,607 operations. Shortened PDS duration for implant surgery from one year to 90 days resulted in $6-14 \%$ of all SSIs being missed. For implant-free procedures, PDS reduction from 30 to 21 days caused similar levels of missed SSIs. In contrast, up to $62 \%$ of SSIs (for cholecystectomy) were missed if other instead of mandatory PDS methods were used. Inferior methods of PDS, rather than shortened PDS durations, may lead to greater underestimation of SSI incidence. Our data validate international recommendations to limit the maximum PDS duration (for implant surgeries) to 90 days for surveillance purposes, as this provides robust insight into trends.

\section{Introduction}

Surgical site infections (SSIs) are a major complication following surgery, causing an important increase in both postoperative morbidity and mortality and healthcare-associated costs [1]. In the Netherlands, SSIs account for about $25 \%$ of healthcare-related infections [2], making them one of the most common nosocomial infections. Cumulative incidences of SSIs (commonly referred to as SSI rates) are considered the primary indicator of the quality of surgical and postoperative care. They are, therefore, an important measure in surveillance systems for healthcare-associated infections.

Identifying SSIs is multidimensional. Case finding using inpatient data may be homogeneous across hospitals: however, focusing only on inpatient data from the initial surgical admission is insufficient [3-5]. As hospital stays have become increasingly shorter, a growing proportion of SSIs is recognised after discharge. Therefore, for measuring SSI incidence, post-discharge surveillance (PDS) has become inevitable. If no PDS is performed, the incidence of SSIs will be greatly underestimated [4-6] and comparisons between hospitals may be flawed. When PDS is performed, two important aspects influence the incidence of SSIs: duration of follow-up and method of follow-up.

Until recently, international consensus was that PDS should be performed up to 30 days, or, if an implant is inserted, one year after the operation $[7,8]$. For reasons of simplicity and to reduce the burden of performing PDS, the United States Centers for Disease Control and Prevention (CDC) in summer 2013 decided to link the duration of PDS to the type of surgery instead of the presence of implants, and to reduce the maximum duration of PDS from one year to 90 days $[9,10]$. Although not officially published yet, for similar reasons, the European Centre for Disease Prevention and Control (ECDC) adopted the 90-day PDS for implant surgeries in 2014 (C. Suetens, personal communication, 15 December 2014). By making these changes, the international consensus on the recommended duration of PDS has been lost and is currently subject to research.

Whereas consensus on the duration of PDS is being sought, there is, however, still no international agreement about the preferred method of PDS. As a result, there is widespread use of various methods that may cause an underestimation of the incidence of SSIs $[3,6,11,12]$. In the Dutch national nosocomial 
Methods of post-discharge surveillance for surgical site infections, the Netherlands, 1999-2008

\begin{tabular}{|c|c|c|c|c|c|c|}
\hline \multirow[b]{2}{*}{ PDS group } & \multirow[b]{2}{*}{ Method of PDS } & \multicolumn{4}{|c|}{ Detection of SSIS } & \multirow{2}{*}{$\begin{array}{c}\text { Mandatory } \\
\text { PDS duration } \\
\text { of } 30 \text { days (no } \\
\text { implant) or one } \\
\text { year (implants) }\end{array}$} \\
\hline & & $\begin{array}{l}\text { During initial } \\
\text { admission }\end{array}$ & $\begin{array}{l}\text { Found by } \\
\text { readmission }\end{array}$ & $\begin{array}{l}\text { Occurring and } \\
\text { treated during } \\
\text { outpatient time }\end{array}$ & $\begin{array}{l}\text { Treated at } \\
\text { a different } \\
\text { facility }\end{array}$ & \\
\hline \multirow{2}{*}{$\begin{array}{l}\text { 'Mandatory' } \\
\text { PDS }^{\text {a }}\end{array}$} & $\begin{array}{l}\text { Registration card in medical records of } \\
\text { all operated patients }\end{array}$ & Yes & Yes & Yes & Yes $^{b}$ & Yes \\
\hline & \begin{tabular}{|l|} 
Retrospective examination of the \\
medical records of all operated patients
\end{tabular} & Yes & Yes & Yes & Yes $^{\mathrm{b}}$ & Yes \\
\hline \multirow{4}{*}{$\begin{array}{l}\text { 'Other' } \\
\text { PDS' }^{c}\end{array}$} & Methods below combined as a group & Mostly $^{d}$ & Mostly $^{d}$ & Sometimes $^{\mathrm{d}}$ & Sometimes $^{d}$ & No \\
\hline & \begin{tabular}{|l|} 
'Passive' PDS: examination of medical \\
records of readmitted patients
\end{tabular} & No & Yes & No & No & No \\
\hline & $\begin{array}{l}\text { Less frequently used 'other' PDS } \\
\text { methods }^{\mathrm{e}}\end{array}$ & Yes $^{\mathrm{d}}$ & $\begin{array}{c}\text { Mostly, } \\
\text { depends on } \\
\text { method used }\end{array}$ & $\begin{array}{l}\text { Depends on } \\
\text { method used }^{d}\end{array}$ & $\begin{array}{l}\text { Depends on } \\
\text { method used }^{d}\end{array}$ & No \\
\hline & No PDS performed & Yes & No & No & No & No \\
\hline
\end{tabular}

SSI: surgical site infection; PDS: post-discharge surveillance; PREZIES: Dutch national nosocomial surveillance network.

a Methods of PDS considered superior and being used mandatorily in PREZIES since 2009.

b Method of case finding not suitable for surgeries exclusively performed by referral hospitals.

All other ways of follow-up used in PREZIES not meeting the criteria for mandatory PDS methods, including performing no PDS.

d Not always reliable.

e For instance, registration card in medical records of a selection of patients, retrospective examination of the medical records of a selection of patients, questionnaires of the patient and/or surgeon, interview of the patient by phone, etc.

surveillance network (PREZIES), several methods of PDS were used until 2009. By 2009, however, two methods for PDS found to be superior, but labour intensive $[3,11]$, became mandatory, as did the duration of PDS: 30 days (non-implant) or one year (implant). These two commitments have led to problems of delayed feedback and increased workload for healthcare professionals. As swift communication of surveillance results after the surgery will help to stimulate healthcare professionals to act and improve, and as the previous international consensus on duration of PDS was lost, the main goal of our study was to investigate the effect of shorter PDS durations on incidence of SSIs, in order to determine a justifiable and advisable duration of PDS. We also aimed to quantify the impact of the mandatory PDS methods and 'other' PDS methods that were acceptable before 2009, in detecting more or less SSIs, and then we compared this impact with the effect of shorter PDS durations on SSI incidences.

\section{Methods}

\section{Design, definitions and data selection}

We used data from PREZIES, the Dutch national nosocomial surveillance network [3]. Hospitals in the Netherlands participate voluntarily in this network and may select surgical procedures for SSI surveillance. PREZIES distinguishes superficial SSIs from deep SSIs, the latter being an umbrella term for so-called deep incisional and organ-space SSIs. In accordance with international guidelines, SSIs were defined as infections that originated within 30 days after surgery (deep and superficial SSIs) or one year after implant surgery (only deep SSIs) [7,8]. An implant is defined as a non-human-derived, implantable foreign body that is permanently placed in a patient during surgery. All SSI surveillance data are collected locally by the hospitals. Further details on PREZIES and data collection, validation, and monitoring quality and reliability have been described previously [3].

SSIs occurring after discharge from hospital were detected by PDS. The operations in the PREZIES database were divided into two groups: those followed up using so-called 'mandatory' PDS methods and all other operations ('other' PDS) (Table 1). The mandatory PDS methods comprise two methods considered superior [11]: (i) use of a registration card; and/or (ii) retrospective examination of medical records for all operated patients. Both have a high sensitivity for capturing SSIs [11] and meet the following five requirements: they detect SSIs during the initial admission, readmission or outpatient time, as well as those treated at a different facility (except for surgeries exclusively performed by referral hospitals) and have a mandatory duration of either 30 days (implant free) or one year (implant used). These methods of PDS were recommended from 1998 to 2008 and became mandatory in 2009. In contrast to operations in the mandatory PDS group, those in the other PDS group were followed up using a variety of PDS methods not fulfilling the mandatory PDS requirements, including using no PDS at all. As such, they differ from the mandatory PDS methods in their way of case finding and/or in their duration. Although their follow-up ranges from no PDS to PDS methods similar to (but not qualifying for) the mandatory methods, the majority of the other PDS group consists of PDS performed by checking records of readmitted patients. 
Details of patients whose operations $(n=105,607)$ were included in the analysis of post-discharge surveillance of surgical site infections, the Netherlands, 1999-2008

\begin{tabular}{|l|c|c|c|c|c|}
\hline Surgical procedure & $\begin{array}{c}\text { Number of } \\
\text { procedures }\end{array}$ & $\begin{array}{c}\text { Mean age in years } \\
\text { (SD) }\end{array}$ & $\begin{array}{c}\text { Mean duration of } \\
\text { surgery in minutes } \\
\text { (SD) }\end{array}$ & $\begin{array}{c}\text { Percentage of men } \\
\text { (n) }\end{array}$ & $\begin{array}{c}\text { Percentage } \\
\text { followed using } \\
\text { mandatory PDS } \\
\text { methods (n) }\end{array}$ \\
\hline Caesarean section & 7,991 & $30.7(5.0)$ & $37.3(20.6)$ & NA & $75(6,007)$ \\
\hline Breast amputation & 5,893 & $60.2(14.1)$ & $74.7(32.8)$ & $1(42)$ & $54(3,165)$ \\
\hline Laparoscopic cholecystectomy & 4,464 & $50.8(15.7)$ & $58.5(36.1)$ & $26(1,176)$ & $36(1,588)$ \\
\hline Colon resection & 5,710 & $67.3(14.0)$ & $113.4(56.4)$ & $48(2,713)$ & $57(3,275)$ \\
\hline Total hip replacement & 49,040 & $69.2(10.5)$ & $74.7(37.3)$ & $31(15,150)$ & $67(33,089)$ \\
\hline Knee replacement & 32,509 & $69.1(9.8)$ & $80.7(40.4)$ & $29(9,415)$ & $66(21,511)$ \\
\hline
\end{tabular}

NA: not applicable; PDS: post-discharge surveillance; SD: standard deviation.

The date of last follow-up was not available from PREZIES data, but it was not necessary as we calculated duration of PDS according to mandatory PDS definitions: 30 days (implant free) or one year (implant used). In case of an SSI, the duration of PDS was the date of infection minus the date of surgery. As we wanted to compare the incidences for the group of other PDS methods from a 30-day or one-year perspective too, the PDS durations were calculated the same way for this group.

We selected data from a 10-year period (1999-2008), with the upper limit chosen to include data from both PDS groups (mandatory and other) for each selected year. Six surgical procedures were chosen for investigation: two procedures with implants (total hip replacement and knee replacement) and four implant-free procedures (breast amputation, colon resection, laparoscopic cholecystectomy and Caesarean section). SSI incidence was determined for each surgical procedure.

\section{Statistical analysis}

We analysed the effect of PDS duration on cumulative incidences of SSIs within both PDS groups using crude SSI incidences that combined deep and superficial SSIs. We plotted cumulative SSI incidences over time for both PDS groups (Kaplan-Meier survival techniques). To be able to compare the effect of better (mandatory) methods with the effect of shorter durations, we calculated crude SSI incidences for each surgical procedure after 90 days, 180 days and one year of PDS for implant procedures, or after 21 days and 30 days of PDS for implant-free surgical procedures. Durations of 21 days and 180 days were arbitrarily chosen but give insight into the timing of infections after surgery. Using the longest PDS duration as a gold standard, we quantified the proportion of SSIs that would be missed by shortening the PDS duration. Finally, for specific time intervals, we calculated the number of detected SSIs as a percentage of the total number at the end of the PDS.
We compared SSI incidences of both PDS groups for each type of surgery by calculating the relative risk of detecting an SSI (hazard ratio (HR)) for other PDS methods compared with mandatory PDS methods, while taking into account varying durations of the PDS (Cox regression analyses). To account for potential confounding (factors associated with the PDS group influencing the HR), we also performed multivariable Cox regression analyses. Several patient-specific and procedurespecific confounders were considered: the American Society of Anesthesiologists score (ASA, a physical status classification system) [13], wound contamination class [14], sex, age, year of surgery (accounting for yearly differences in hospitals' participation, with their differences in methods of PDS used) and duration of surgery. The potential confounders (determinants) were tested for their impact on the regression coefficient. A determinant altering the coefficient of the (univariate) analysis by $10 \%$ or more was considered a confounder and was included in the multivariable model by manual forward inclusion. This procedure was repeated for other potential confounders until the final model was constructed. For each type of surgery, we converted the resulting HRs into the proportion of SSIs that would be missed when choosing other instead of mandatory, methods of PDS (proportion missed $=(1-H R) \times 100$ ). Again, all analyses were performed at 21 days and 30 days (implant-free procedures) or at 90 days, 180 days and one year (implant procedures). Most Cox regression analyses were performed for deep and superficial SSIs, both in combination and separately.

Statistical significance was defined at 0.05 , and a power of $80 \%$ was chosen. All statistical procedures were performed with SAS software, version 9.3 (SAS institute).

\section{Results}

Data selection

From 1999 to 2008, PREZIES collected data on 234,841 surgical procedures. For the six surgical 


\section{FIGURE 1}

Cumulative incidence of surgical site infections, the Netherlands, 1999-2008 ( $\mathrm{n}=105,607)$

A. Caesarean section

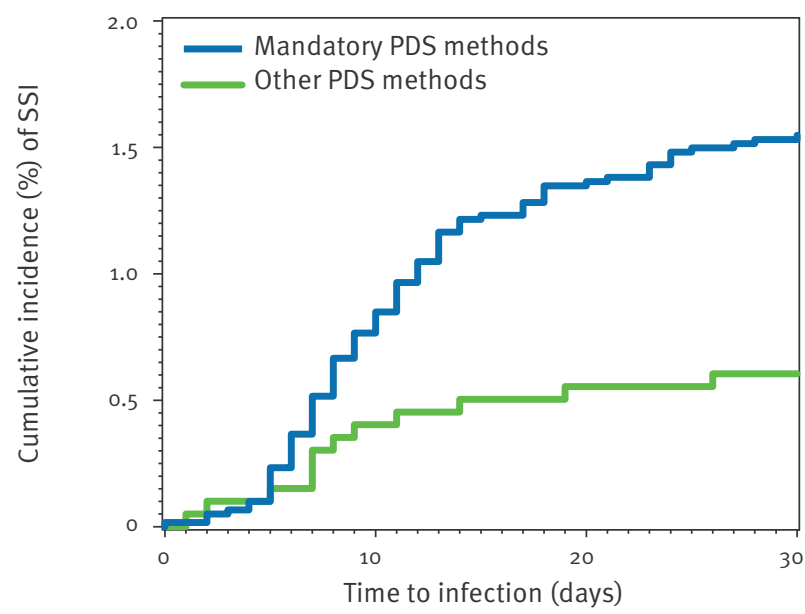

C. Laparoscopic cholecystectomy

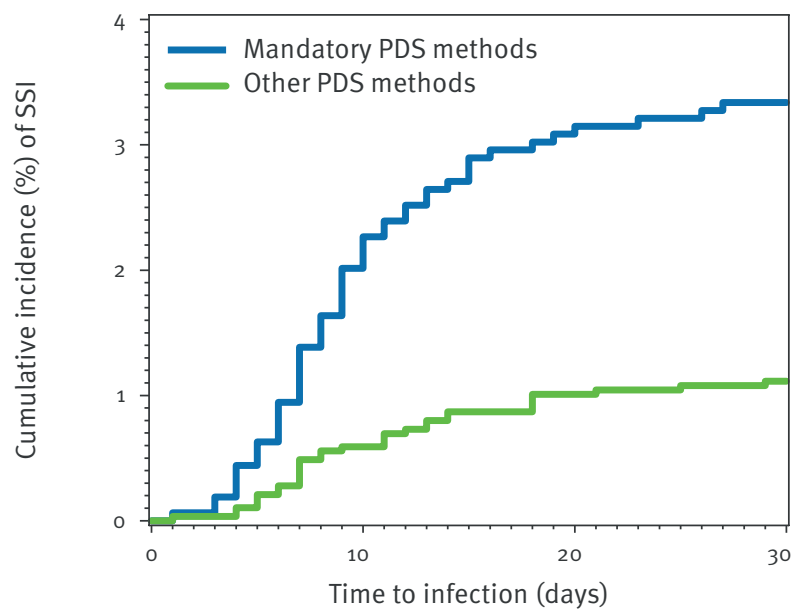

E. Total hip replacement

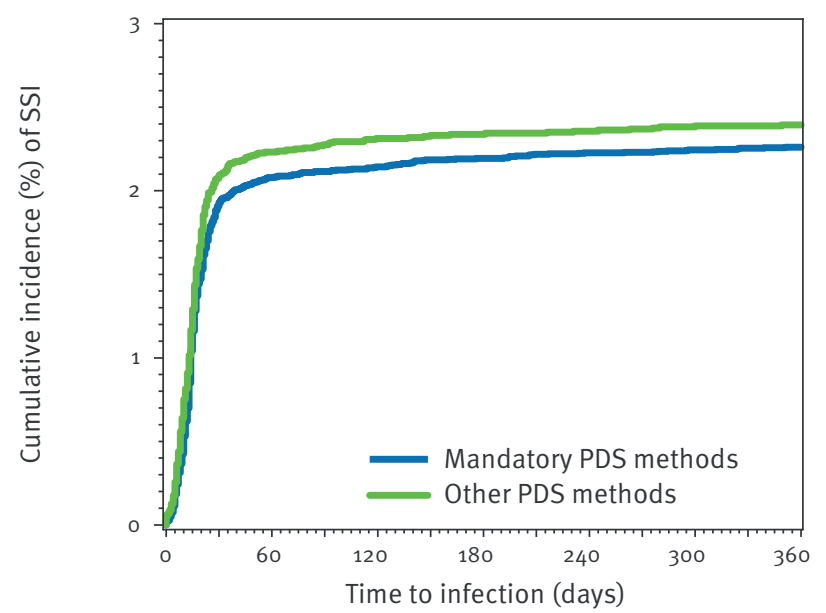

B. Breast amputation

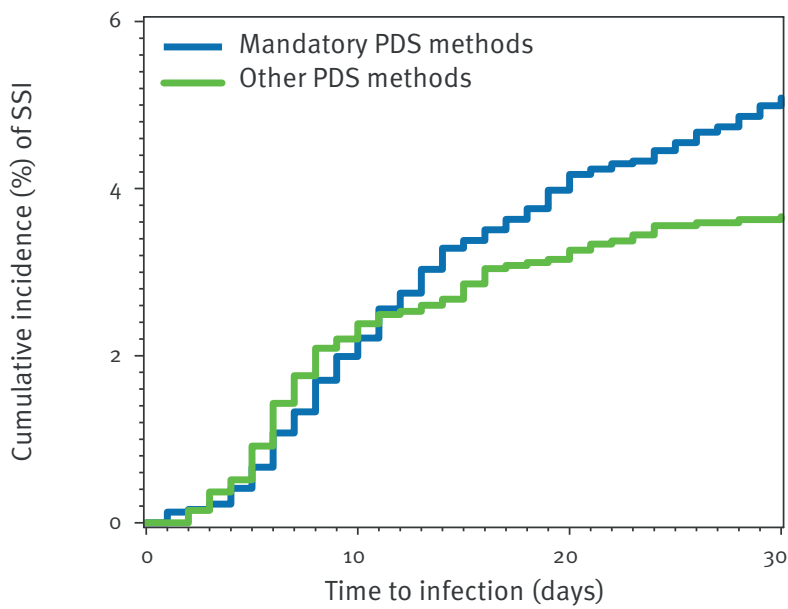

D. Colon resection

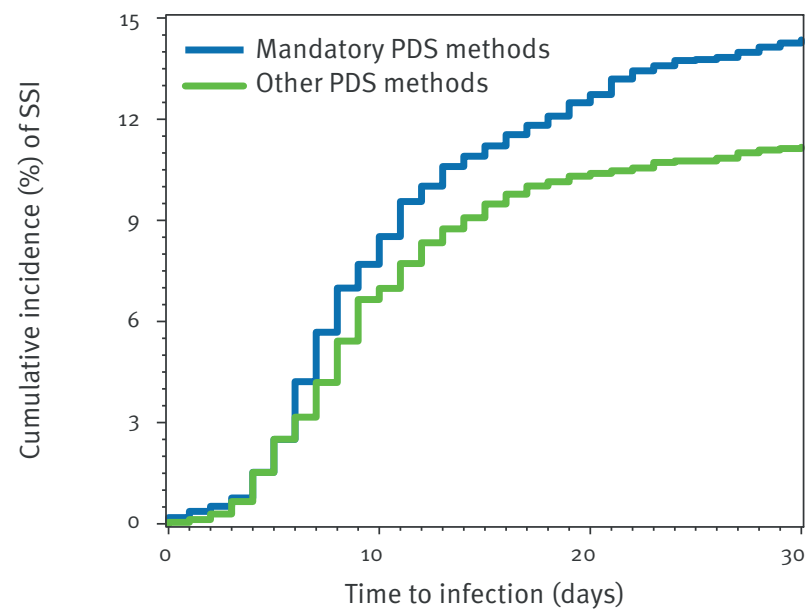

F. Knee replacement

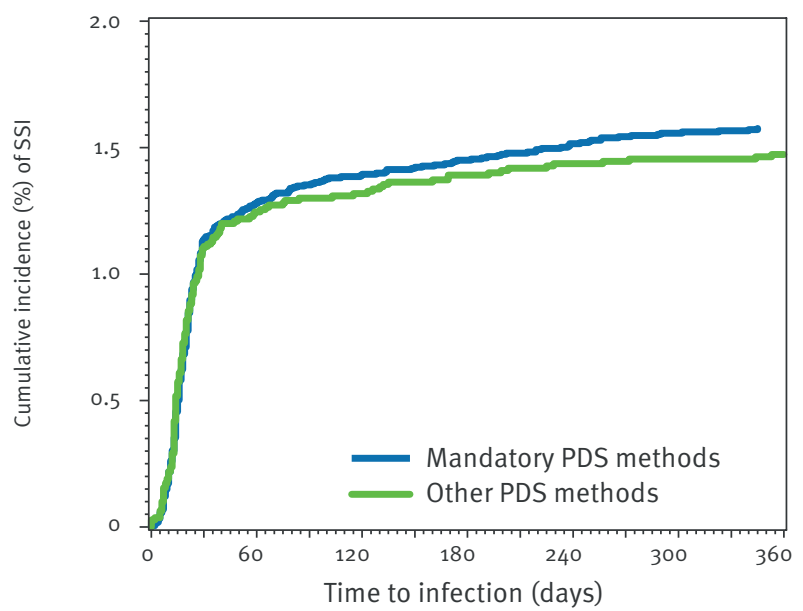

SSI: surgical site infection; PDS: post-discharge surveillance; PREZIES: Dutch national nosocomial surveillance network.

Crude cumulative SSI incidence is shown over time for the 'mandatory' methods of PDS (blue line) and 'other' methods of PDS (green line) for the six surgical procedures. Mandatory PDS methods are considered superior and have been mandatory in PREZIES since 2009. 'Other' PDS methods are all other methods used in the PREZIES surveillance of SSIs not meeting the criteria for mandatory PDS methods.

Deep and superficial SSIs were combined. For the two procedures with implants (knee and total hip replacement), the maximum PDS duration is one year. For the other four procedures, the maximum PDS duration is 30 days. 


\section{FIGURE 2}

Distribution of site infections detected by post-discharge surveillance, by time interval, the Netherlands, 1999-2008 $(\mathrm{n}=68,635)$

A. Surgery without implants: Caesarean section, breast amputation, laparoscopic cholecystectomy and colon resection

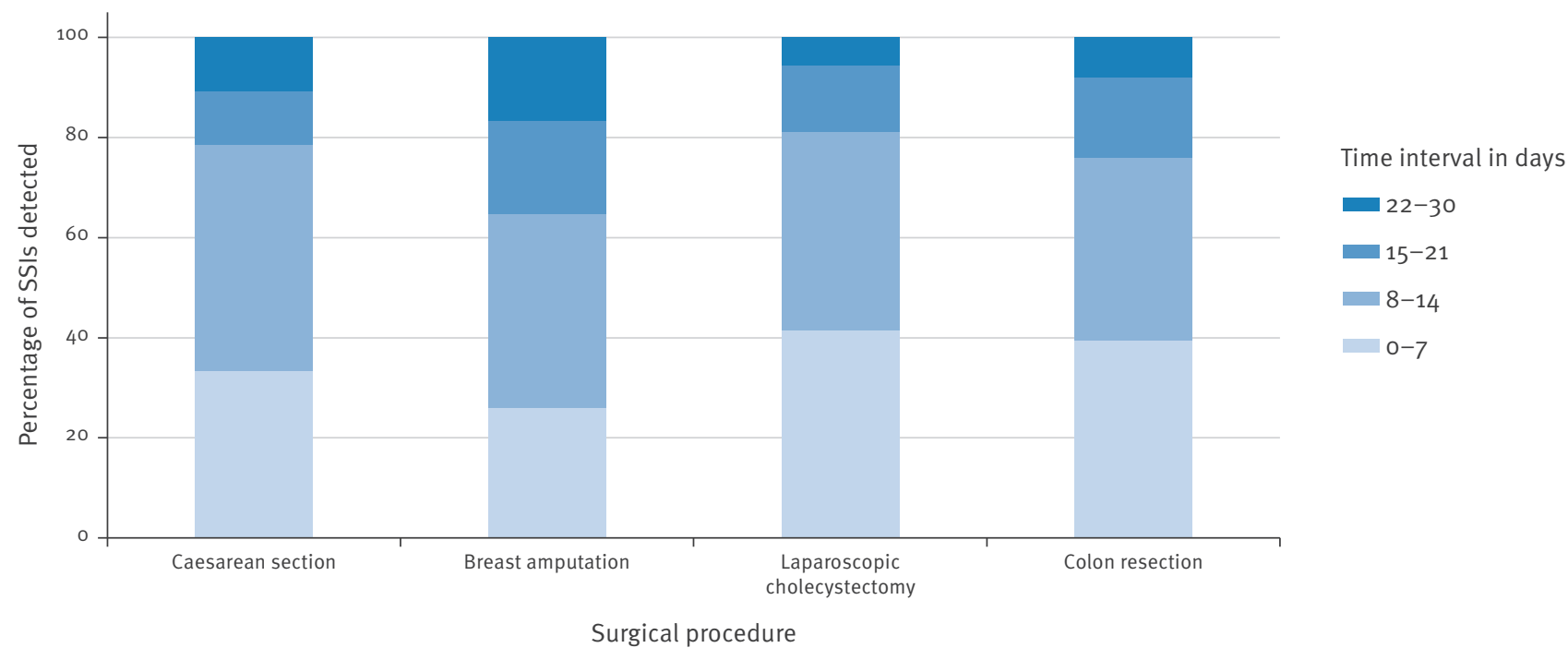

B. Surgery with implants: total hip replacement and knee replacement

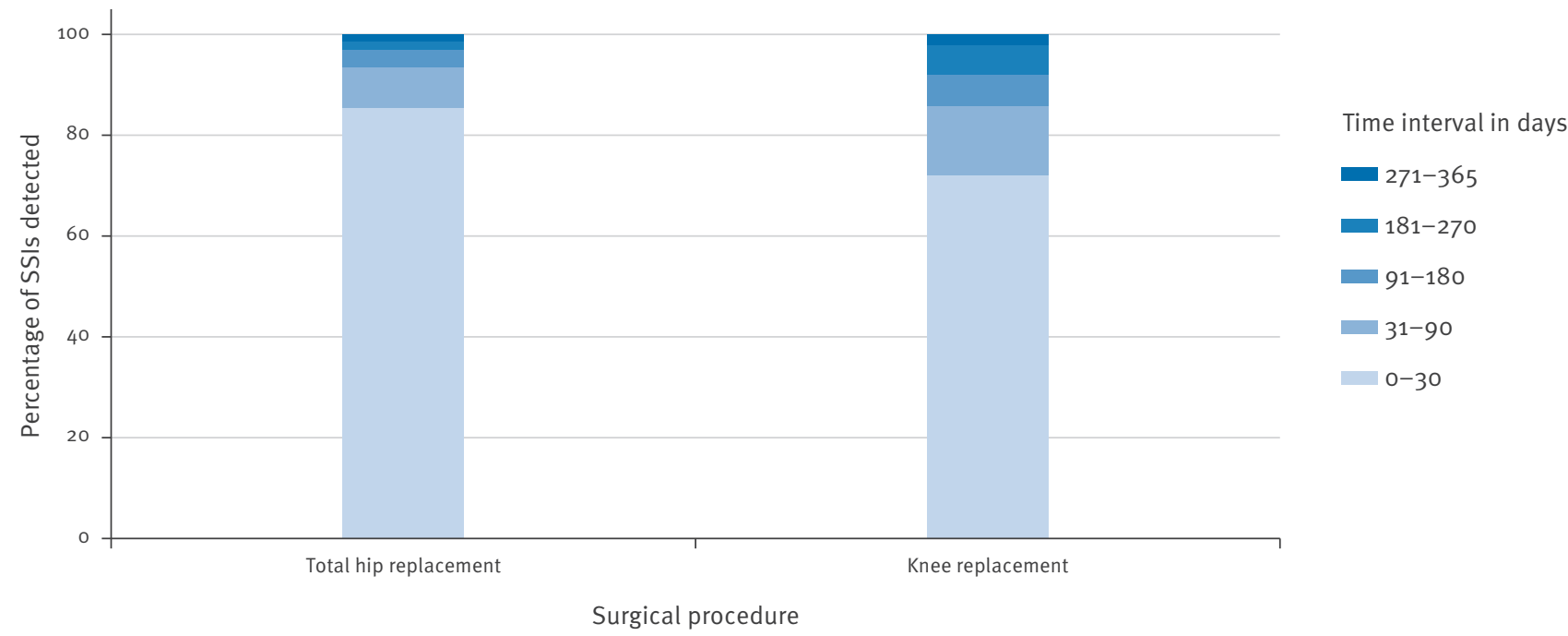

SSI: surgical site infection; PDS: post-discharge surveillance; PREZIES: Dutch national nosocomial surveillance network.

The proportion of SSIs detected during each PDS time interval is displayed as a percentage of the total number of SSIs detected. Only SSIs detected by the 'mandatory' methods of PDS are presented; deep and superficial SSIs combined. For the four procedures without implants (panel A) the PDS duration (30 days) is divided into four intervals; for the two procedures with implants (panel B) the PDS duration of one year) is divided into five intervals.

Mandatory PDS methods are considered superior and have been mandatory in PREZIES since 2009. 


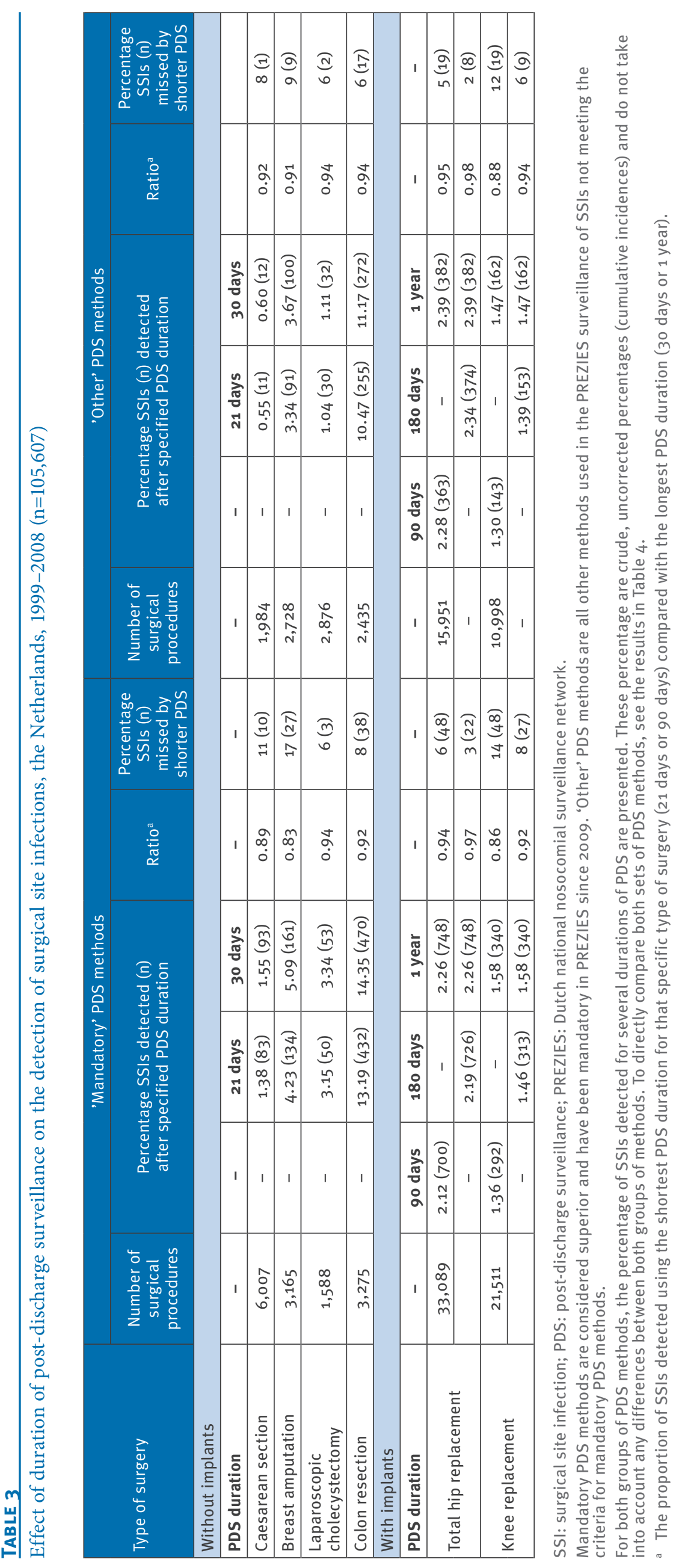


Comparison of methods of post-discharge surveillance on the detection of surgical site infections, the Netherlands, 1999-2008 $(n=105,607)$

\begin{tabular}{|c|c|c|c|c|}
\hline \multirow{2}{*}{ Type of surgery } & \multirow{2}{*}{ Duration of PDS } & \multicolumn{3}{|c|}{$\begin{array}{l}\text { Detection of SSIs by 'other' PDS methods } \\
\text { compared with 'mandatory' PDS methods }\end{array}$} \\
\hline & & Hazard ratio $(95 \% \mathrm{Cl})$ & Variables adjusted for & $\begin{array}{l}\text { Percentage SSIs missed } \\
\text { by 'other' methods }\end{array}$ \\
\hline Caesarean section & 30 days & $0.39^{\mathrm{a}}(0.21-0.71)$ & $N A^{a}$ & 61 \\
\hline Breast amputation & 30 days & $0.59(0.43-0.81)$ & $Y$ & 41 \\
\hline Laparoscopic cholecystectomy & 30 days & $0.38(0.23-0.63)$ & $\mathrm{Y}$ & 62 \\
\hline Colon resection & 30 days & $0.91(0.77-1.07)$ & $\mathrm{Y}, \mathrm{A}, \mathrm{W}$ & 9 \\
\hline \multirow{2}{*}{ Total hip replacement } & 90 days & $1.06(0.92-1.21)$ & $\mathrm{Y}, \mathrm{A}, \mathrm{Ag}, \mathrm{D}$ & -6 \\
\hline & 1 year & $1.04(0.92-1.19)$ & $\mathrm{Y}, \mathrm{A}, \mathrm{Ag}, \mathrm{D}$ & -4 \\
\hline \multirow{2}{*}{ Knee replacement } & 90 days & $0.79(0.64-0.98)$ & $\mathrm{Y}, \mathrm{A}$ & 21 \\
\hline & 1 year & $0.78(0.64-0.95)$ & $\mathrm{Y}, \mathrm{A}$ & 22 \\
\hline
\end{tabular}

A: American Society of Anesthesiologists (ASA) score [13]; Ag: age; Cl: confidence interval; D: duration of surgery; NA: not applicable; PDS: post-discharge surveillance; PREZIES: Dutch national nosocomial surveillance network; SSI: surgical site infection; W: wound class [14]; Y: year of surgery.

Mandatory PDS methods are considered superior and have been mandatory in PREZIES since 2009. 'Other' PDS methods are all other methods used in the PREZIES surveillance of SSIs not meeting the criteria for mandatory PDS methods.

a No confounding detected; univariate hazard ratio presented.

procedures under investigation, data on 127,705 surgeries was available; 7,000 were excluded because it was unknown whether an implant was used. Another 11,819 records were excluded because either no use of implants was registered (knee replacement and total hip replacement) or use of implants was registered in a predominantly implant-free procedure (breast amputation, colon resection, laparoscopic cholecystectomy or Caesarean section). Finally, 3,279 surgical procedures were excluded because the method of PDS was unknown. Therefore, our results are based on data from 105,607 operations, which were collected at 87 hospital sites in the Netherlands. The patient characteristics are described in Table 2.

\section{Effect of post-discharge surveillance duration on incidence of surgical site infections}

Cumulative SSI incidences over time for both PDS groups for the six surgical procedures show that most SSIs were detected in the first weeks and months of follow-up (Figure 1).

The distribution of SSIs detected per time interval as a percentage of the total number of SSIs detected with the mandatory methods of PDS is shown in Figure 2. An additional $6 \%$ (3/53, cholecystectomy) to $17 \%(27 / 161$, breast amputation) of all SSIs were detected in the final nine days of a 30-day follow-up (Figure 2, Table 3). Furthermore, 94\% (700/748, hip) and 86\% (292/340, knee) of all SSIs were already detected after 90 days of a one-year follow-up. After 180 days of follow-up, $97 \%(726 / 748)$ and $92 \%$ (313/340), respectively, were detected. The same analyses for the other methods of PDS yielded comparable results (Table 3 ).
Impact of post-discharge surveillance method on incidence of surgical site infections

Our results show an important difference between both PDS groups regarding the percentage of SSIs detected (Figure 1, Table 3). Crude SSI incidences at the end of PDS were lower for the other PDS methods (0.60\% $(12 / 1,984)$ to $11.17 \%(272 / 2,435))$ than for the mandatory PDS methods $(1.55 \%(93 / 6,007)$ to $14.35 \%$ $(470 / 3,275))$, except for hip replacements.

The HRs comparing the SSI incidences of other PDS methods with those of mandatory PDS methods, while accounting for differences between both groups, confirm a lower chance of detecting SSIs by other methods of PDS in five of the six types of surgery (statistically significant for four of the procedures) (Table 4). Analyses for a PDS duration of 21 days (implant-free procedures) and 180 days (knee replacement and total hip replacements) yielded similar results (data not shown).

Up to $62 \%$ of all SSIs (for cholecystectomy) may be missed during the surveillance when other methods of PDS are used instead of the mandatory methods. Additional analysis into impact of PDS methods on deep vs superficial SSI incidences indicated that in colon resection, it was only superficial (and not deep) SSI incidence that dropped when other instead of mandatory methods of PDS were used (HR: $0.74 ; 95 \% \mathrm{Cl}$ : $0.57-0.96)$. For the remaining four types of surgery showing a decreased SSI incidence when other PDS methods were used, the incidence of superficial SSIs as well as deep SSIs dropped, although the latter especially was not always statistically significant. HRs and $95 \% \mathrm{Cls}$ for superficial and deep SSIs respectively were: Caesarean section 0.35 (0.21-0.70, superficial) 
and 0.64 (0.18-2.34, deep); breast amputation 0.50 (0.34-0.73) and 0.84 (0.49-1.45); laparoscopic cholecystectomy $0.13(0.06-0.27)$ and 0.85 (0.43-1.69); and knee replacement $0.73(0.54-1.00)$ and 0.75 (0.58-0.98).

\section{Discussion}

\section{Duration of post-discharge surveillance}

Although the cumulative incidence of SSIs varied greatly between procedures, the number of new SSIs detected decreased during the PDS for all types of surgery; SSIs were detected primarily in the first weeks or months of the surveillance. A reduction in the PDS duration for implant procedures from one year to 90 days would potentially miss only $6 \%$ (hip replacement) and $14 \%$ (knee replacement) of SSIs. This would result in a decrease of the SSI incidence of $0.14 \%$ (from $2.26 \%$ to $2.12 \%$ ) and $0.22 \%$ (from $1.58 \%$ to $1.36 \%$ ) respectively, meaning that for every 714 hip or 455 knee replacements, one SSI would be missed. In other words, shortening the duration of PDS by nine months would not cause a substantial drop in SSI incidence. When the aim is to report SSIs for clinical purposes, missing even a small proportion of SSIs might be unacceptable. For surveillance purposes, however, not only the reliability of the SSI incidence but also the workload involved in PDS and the speed of feedback to the healthcare professional must be considered. It is acceptable for surveillance of SSIs to underestimate actual SSI incidence, as long there are other important advantages of the surveillance. The advantages of a shorter PDS seem not to outweigh the effect on the SSI incidence for implant-free surgeries. After all, up to $17 \%$ (breast amputation) of the identified SSIs were detected in the final period (days 22-30), and these additional nine days of PDS would not have a considerable impact on workload or swiftness of the feedback. For implant procedures, however, considering all the effort required to perform a one-year-long PDS and the advisability of returning surveillance results to healthcare professionals sooner rather than later, it would be worthwhile to shorten the recommended PDS duration.

\section{Methods of post-discharge surveillance}

SSI incidence varied between the types of procedure, but there was also a great variation in SSI incidence between the two groups of PDS methods. We found that the chances of detecting SSIs in implant-free procedures were lower (HRs varying from $0.38(95 \% \mathrm{Cl}$ : $0.23-0.63)$ to $0.91(95 \% \mathrm{Cl}: 0.77-1.07)$ when other, instead of mandatory, PDS methods were used. For implant surgery, the results were less straightforward. The crude SSI incidence did not differ significantly between the other and mandatory methods of PDS. When adjusted for available confounders, however, the mandatory methods of PDS again resulted in significantly improved detection of SSIs for knee-replacement surgery (HR: 0.79 ( $95 \% \mathrm{Cl}: 0.64-0.98)$ ). There may be several reasons why the mandatory PDS methods were not more sensitive for hip replacements. Firstly, due to the severe complications of a deep SSI in the hip joint, patients with deep SSIs following hip replacement are always readmitted. This makes other methods of PDS such as 'only checking readmitted patients' more sensitive for hip replacement surgery than for procedures with less severe SSIs. Another explanation could be our observation that the other PDS methods used for the surveillance of hip- (and to a lesser degree, knee-) replacement surgery were in general more similar to the mandatory methods of PDS than the other PDS methods used following other types of surgery.

In general, the decrease in detection of superficial SSIs by other methods was more noticeable than that of deep SSIs. The better detection of SSIs using mandatory methods of PDS can be explained by the fact that the these methods obviously aim at finding cases during more stages (during initial admission, readmission, outpatient time, and for those treated in another hospital) and does so for a mandatory period (30 days or one year). In addition, since superficial SSIs do not always require readmission and thus are more easily missed using 'other' PDS methods, the better detection of SSIs by mandatory PDS was logically more marked for superficial SSIs than for deep SSIs.

The Dutch mandatory methods of PDS are considered labour intensive due to the use of specific case-finding methods for several stages during the (mandatory) long period. Especially for PDS durations of one year, the surveillance work is generally carried out twice for each operated patient (after one to three months, and again after a year) or more frequently. Nevertheless, when compared with other methods of PDS, the increased detection of SSIs (up to $62 \%$ for cholecystectomy) justifies the use of the mandatory methods. We are convinced that the costs and time saved by shortening the mandatory PDS durations from one year to 90 days can be applied to improve and intensify existing methods of PDS.

\section{Comparison with literature}

Our study focused on the effect of both PDS duration and PDS method on SSI incidence and on the timing (accumulation) of SSIs. To the best of our knowledge, no European studies and only a few American and Canadian studies have analysed timing of SSIs to quantify the impact of a shorter PDS duration [10,1518]. None of these studies used survival techniques to visually demonstrate the accumulation of SSIs over time. Also, we are not aware of any studies in which multivariable Cox regression models were used to analyse the impact of method of PDS on incidence of SSIs.

Our results are in line with other studies investigating the effect of duration of PDS on SSI incidence. The vast majority of SSIs were detected within a 90-day window [10,15-18], varying from about $70 \%$ [10] to $100 \%$ [17]. After 90 days, only a few more SSIs were detected, triggering discussion about whether those late SSIs are truly due to preventable issues during the 
operation [16]. Three of the five studies mentioned [1517] confirm our finding that more SSIs are missed after knee-replacement surgery than after hip replacement surgery when the follow-up is reduced to 90 days.

Regarding the impact of different methods of PDS on SSI incidence, some studies have tried to compare the results of different methods of PDS $[3,4,6,12,19,20]$. Similar to our results, most conclude that enhanced efforts to perform PDS result in an improved detection of deep and (more markedly) superficial SSIs $[3,4,19,20]$. Thus, hospitals using improved surveillance methods will be 'penalised' with a higher incidence of SSIs, especially superficial SSIs.

To prevent hospitals being penalised in this way, Wilson et al. propose that the use of in-hospital incidence density (number of SSIs per 1,000 post-operative inpatient days) might be a more reliable indicator than cumulative incidence for comparison between hospitals or countries [21]. After all, in-hospital case finding is probably more homogeneous across hospitals than post-discharge case finding, and, by focusing on inpatient data alone, differences in methods of PDS do not influence the indicator. By focusing on inpatient data, however, the differences in post-operative hospital stay largely influence the number of SSIs detected. When the cumulative incidence is linearly related to length of time after surgery, the incidence density adequately adjusts for these differences in post-operative hospital stay. However, as we have shown in our analysis, the cumulative incidence is not a linear function of time after surgery, but has an S-shaped curve (Figure 1). As a result, calculations of in-hospital incidence density depend on the duration of post-operative hospital stay. During the first 10-14 days after surgery, a longer hospital stay leads to a higher incidence density (slope of line drawn from origin 0 to a point on the steep part of the curve). After that, however, the incidence density will decrease again (slope of a line drawn from origin 0 to a point on the flattened right part of the curve). Thus, even with perfect detection of SSIs, the in-hospital incidence density depends on the average duration of admission after surgery, and will be progressively underestimated as the time after surgery increases beyond two weeks. Therefore, we are convinced that a fixed, mandatory duration of following patients after surgery should be recommended to keep data comparable. The second-best option, if durations of follow-up do differ, the use of incidence densities could be considered but certainly has many limitations.

\section{Strengths and limitations of our study}

The strength of our study is that we gained insight into cumulative SSI incidences over time using survival techniques. Additionally, besides comparing crude SSI incidences, we used multivariable regression techniques to compare both groups of PDS methods, which allowed for correction for possible confounders. The correction, however, was probably not complete, resulting in some residual confounding in our analyses. For instance, since method of PDS was obviously not randomised within the hospitals, we corrected for differences in PDS by using 'year of surgery' as a proxy (to account for yearly differences in hospitals' participation with their differences in methods of PDS used). Since we used a proxy, this correction is probably incomplete. On the other hand, it seems unlikely that our results can be explained by hospitals with lower 'true' SSI incidences systematically choosing other methods of PDS instead of mandatory methods. We would rather expect the opposite, which would result in an underestimation of the effect found in this study. Finally, we are convinced that by using surveillance data, we studied daily practice; therefore, the study itself caused no selection or changes in professional behaviour for the detection of SSIs.

A potential weakness is the fact that sometimes there was not enough power to significantly identify (small) differences, especially for surgical procedures with a low SSI incidence, or for procedures with a relatively small number of operations, or both. Another point is that we included two orthopaedic procedures for the implant surgeries; we could not include other implant procedures (for example, breast-enlargement surgery) because not enough data were available. Also, the Dutch mandatory methods of PDS are not an international gold standard for detecting SSIs, and the analysed group of other PDS methods is a collection of several different methods of PDS. And finally, since durations for mandatory PDS were a mandatory 30 days or one year, we accordingly assumed all followup durations in absence of an SSI to be 30 days or one year. If there were any records in the mandatory PDS group that did not completely comply with the protocol for mandatory PDS, this assumption may have been incorrect. This may have caused an underestimation of SSI incidence for the mandatory PDS group, making the detected differences between both PDS groups smaller. However, using the experience from our visits to the hospitals (as part of our quality assurance system) [22], we are of the opinion that this effect is either non-existent or negligible.

In the Dutch surveillance network, many hospitals chose knee- or hip-replacement surgeries for their surveillance of SSIs. From our visits to the hospitals [22], we noticed that the other PDS methods for these surgeries were often more similar to the mandatory PDS methods (and hence of higher quality regarding detection of SSIs) than those for procedures less frequently included in the surveillance. Therefore, the results of comparing both PDS groups for hip replacements and knee replacements may not simply be generalised to all implant surgeries. However, the effect of shortening the PDS duration did not differ between both groups of PDS methods. We, therefore, hypothesise that our conclusions and advice regarding shortening the PDS duration for knee replacements and total hip replacements may be generalised to other implant procedures. Nevertheless, we consider it prudent to perform similar 
analyses for other implant procedures to confirm our results.

\section{Recent developments and implications}

In July 2013, the United States CDC's National Healthcare Safety Network (NHSN) reduced the maximum PDS duration from one year to 90 days $[9,10]$. This change was made on the recommendation of an SSI surveillance working group (CDC working with clinical partners) that was supported by the Healthcare Infection Control Practices Advisory Committee (HICPAC). Reasons for changing the PDS duration were that 'The benefits include simplicity, a shorter followup time for many procedures that will reduce burden, and an opportunity to intensify post-discharge surveillance efforts for a shorter follow-up period' [10] and the data presented to support the NHSN decision [10,1618] are in line with our results. Another advantage of a shorter PDS duration could be that those hospitals and countries currently investing more time and energy than others in the final nine months of surveillance will no longer be 'penalised' with higher SSI incidences for their efforts. This will make inter-hospital or intercountry comparisons of SSI incidences more valuable, although ranking of hospitals or countries based on SSI incidence should be avoided [23].

\section{Conclusion and recommendation}

A one-year PDS for hip- and knee-replacement surgery no longer seems justified, since a 90-day PDS would capture the majority of the SSIs equally as well. Maintaining a PDS duration of 30 days for implant-free surgeries, however, is still recommended.

Although a small proportion of the SSIs would be missed for implant procedures, shortening the duration of PDS to 90 days would substantially facilitate prompt feedback to healthcare professionals and reduce workload for those performing the surveillance. Also, we conclude that choosing a method of PDS superior in detecting SSIs (such as the Dutch mandatory methods) is at least as important as choosing a sensible duration of PDS, because inferior methods of PDS may lead to greater underestimation of SSI incidence than shorter PDS durations do.

Our data validate international recommendations to limit the duration of PDS for implant surgeries to 90 days for surveillance purposes, as this provides robust insight into trends. Costs and time saved by shortening the duration of PDS can be applied to improve the methods of PDS.

\section{Acknowledgements}

The authors wish to thank all infection control practitioners, microbiologists, and other hospital staff from the participating hospitals who contributed to this study. We also thank Jeroen Alblas for editing the figures, Sabine de Greeff and Titia Hopmans for carefully reading the manuscript, and Sally Ebeling for English revision.
Conflict of interest

None declared.

Authors' contributions

M. Koek designed the study, conducted the analyses and wrote the manuscript. B. van Benthem helped designing the study's analytic strategy and supervised the whole research process. J. Wille, M. Isken and A. Voss helped writing and reviewing the manuscript.

\section{References}

1. de Lissovoy G, Fraeman K, Hutchins V, Murphy D, Song D, Vaughn BB. Surgical site infection: incidence and impact on hospital utilization and treatment costs. Am I Infect Control. 2009;37(5):387-97. http://dx.doi.org/10.1016/j. ajic.2008.12.010 PMID:19398246

2. van der Kooi TI, Manniën J, Wille JC, van Benthem BH. Prevalence of nosocomial infections in The Netherlands, 2007-2008: results of the first four national studies. J Hosp Infect. 2010;75(3):168-72. http://dx.doi.org/10.1016/j. jhin.2009.11.020 PMID:20381910

3. Manniën J, Wille JC, Snoeren RL, van den Hof S. Impact of postdischarge surveillance on surgical site infection rates for several surgical procedures: results from the nosocomial surveillance network in The Netherlands. Infect Control Hosp Epidemiol. 2006;27(8):809-16. http://dx.doi. org/10.1086/506403 PMID:16874640

4. Tanner J, Padley W, Kiernan M, Leaper D, Norrie P, Baggott R. A benchmark too far: findings from a national survey of surgical site infection surveillance. J Hosp Infect. 2013;83(2):87-91. http://dx.doi.org/10.1016/j.jhin.2012.11.010 PMID:23332352

5. Calderwood MS, Kleinman K, Bratzler DW, Ma A, Bruce CB, Kaganov RE, et al.; Centers for Disease Control and Prevention Epicenters Program; Oklahoma Foundation for Medical Quality. Use of Medicare claims to identify US hospitals with a high rate of surgical site infection after hip arthroplasty. Infect Control Hosp Epidemiol. 2013;34(1):31-9. http://dx.doi. org/10.1086/668785 PMID:23221190

6. Reilly J, Allardice G, Bruce J, Hill R, McCoubrey J. Procedurespecific surgical site infection rates and postdischarge surveillance in Scotland. Infect Control Hosp Epidemiol. 2006;27(12):1318-23. http://dx.doi.org/10.1086/509839 PMID:17152029

7. Horan TC, Andrus M, Dudeck MA. CDC/NHSN surveillance definition of health care-associated infection and criteria for specific types of infections in the acute care setting. Am J Infect Control. 2008;36(5):309-32. http://dx.doi.org/10.1016/j. ajic.2008.03.002 PMID:18538699

8. European Centre for Disease Prevention and Control (ECDC). Surveillance of surgical site infections in European hospitals - HAISSI protocol. Version 1.02. Stockholm: ECDC; 2012. Available from: http://www.ecdc.europa.eu/en/publications/ Publications/120215_TED_SSI_protocol.pdf

9. Centers for Disease Control and Prevention (CDC)/National Healthcare Safety Network (NHSN). Surgical site infection (SSI) event. Procedure-associated module SSI. Jan 2015. Atlanta, GA: CDC. [Accessed 5 Feb 2015]. Available from: http://www. cdc.gov/nhsn/PDFs/pscManual/9pscSSIcurrent.pdf

10. Healthcare Infection Control Practices Advisory Committe (HICPAC). Meeting summary report. June 14-15, 2012, Atlanta, GA. Atlanta, GA: HICPAC, Centers for Disease Control and Prevention. [Accessed 19 Feb 2015]. Available from: http:// www.cdc.gov/hicpac/pdf/mm/HICPAC-MEETING-MINUTESJUNE-2012.pdf

11. de Haas R, Mintjes-de Groot AJ, Geubbels ELPE, van den Berg JMJ, de Boer AS. Inventarisatie van surveillance na ontslag in het PREZIES-project. [Inventory of post discharge surveillance in the PREZIES-network]. Bilthoven: National Institute for Public Health and the Environment (RIVM)/Utrecht: Dutch Institute for Healthcare Improvement (CBO); 1998. Dutch. Available from: http://www.rivm.nl/dsresource?objectid=rivmp :256406\&type $=$ org\&disposition=inline\&ns_nc=1

12. Petherick ES, Dalton JE, Moore PJ, Cullum N. Methods for identifying surgical wound infection after discharge from hospital: a systematic review. BMC Infect Dis. 2006;6(1):170. http://dx.doi.org/10.1186/1471-2334-6-170 PMID:17129368

13. Owens WD, Felts JA, Spitznagel EL Jr. ASA physical status classifications: a study of consistency of ratings. Anesthesiology. 1978;49(4):239-43. http://dx.doi. org/10.1097/00000542-197810000-00003 PMID:697077 
14. Mangram AJ, Horan TC, Pearson ML, Silver LC, Jarvis WR; Centers for Disease Control and Prevention (CDC) Hospital Infection Control Practices Advisory Committee. Guideline for Prevention of Surgical Site Infection, 1999. Am J Infect Control. 1999;27(2):97-134. http://dx.doi.org/10.1016/So1966553(99)70088-X PMID:10196487

15. Dicks KV, Lewis SS, Durkin MJ, Baker AW, Moehring RW, Chen LF, et al. Surveying the surveillance: surgical site infections excluded by the January 2013 updated surveillance definitions. Infect Control Hosp Epidemiol. 2014;35(5):570-3. http://dx.doi. org/10.1086/675837 PMID:24709727

16. Yokoe DS, Avery TR, Platt R, Huang SS. Reporting surgical site infections following total hip and knee arthroplasty: impact of limiting surveillance to the operative hospital. Clin Infect Dis. 2013;57(9):1282-8. http://dx.doi.org/10.1093/cid/cit516 PMID:23912846

17. Lankiewicz JD, Yokoe DS, Olsen MA, Onufrak F, Fraser VJ, Stevenson K, et al. Beyond 30 days: does limiting the duration of surgical site infection follow-up limit detection? Infect Control Hosp Epidemiol. 2012;33(2):202-4 . http://dx.doi. org/10.1086/663715 PMID:22227993

18. Bryce E, Forrester L. How long is long enough? Determining the optimal surgical site infection surveillance period. Infect Control Hosp Epidemiol. 2012;33(11):1178-9. http://dx.doi. org/10.1086/668037 PMID:23041824

19. Calderwood MS, Ma A, Khan YM, Olsen MA, Bratzler DW, Yokoe DS, et al. Use of Medicare diagnosis and procedure codes to improve detection of surgical site infections following hip arthroplasty, knee arthroplasty, and vascular surgery. Infect Control Hosp Epidemiol. 2012;33(1):40-9. http://dx.doi. org/10.1086/663207 PMID:22173521

20. Ming DY, Chen LF, Miller BA, Anderson DJ. The impact of depth of infection and postdischarge surveillance on rate of surgicalsite infections in a network of community hospitals. Infect Control Hosp Epidemiol. 2012;33(3):276-82. http://dx.doi. org/10.1086/664053 PMID:22314065

21. Wilson J, Ramboer I, Suetens C; HELICS-SSI working group. Hospitals in Europe Link for Infection Control through Surveillance (HELICS). Inter-country comparison of rates of surgical site infection--opportunities and limitations. J Hosp Infect. 2007;65(Suppl 2):165-70. http://dx.doi.org/10.1016/ S0195-6701(07)60037-1 PMID:17540264

22. Manniën J, van der Zeeuw AE, Wille JC, van den Hof S. Validation of surgical site infection surveillance in the Netherlands. Infect Control Hosp Epidemiol. 2007;28(1):36-41. http://dx.doi.org/10.1086/509847 PMID:17230385

23. van Dishoeck AM, Koek MB, Steyerberg EW, van Benthem BH, Vos MC, Lingsma HF. Use of surgical-site infection rates to rank hospital performance across several types of surgery. $\mathrm{Br}$ J Surg. 2013;100(5):628-37. http://dx.doi.org/10.1002/bjs.9039 PMID:23338243 